\title{
Prediction Score-Guided Genetic Testing for Hypertrophic Cardiomyopathy
}

\author{
Kaoru Ito, MD, PhD; Hiroyuki Morita, MD, PhD
}

H ypertrophic cardiomyopathy (HCM) is one of the most common inherited cardiac diseases. Whenever we perform genetic testing to identify a causative genetic variant for HCM, we should consider the cost and the benefits for patients and their family members. Because genetic testing is costly for the patient and requires the cooperation of clinical geneticists and genetic consultants for the care of patients as well as their family members, there is a need for indicators of how positively genetic

\section{Article p 669}

testing should be performed in clinical practice. Practically, other factors, such as hypertension and stenotic valvular heart diseases, can also induce HCM-like cardiac manifestations, so it is often hard to define the appropriate target patients for genetic testing. According to past literature, genetic testing for patients clinically diagnosed with HCM

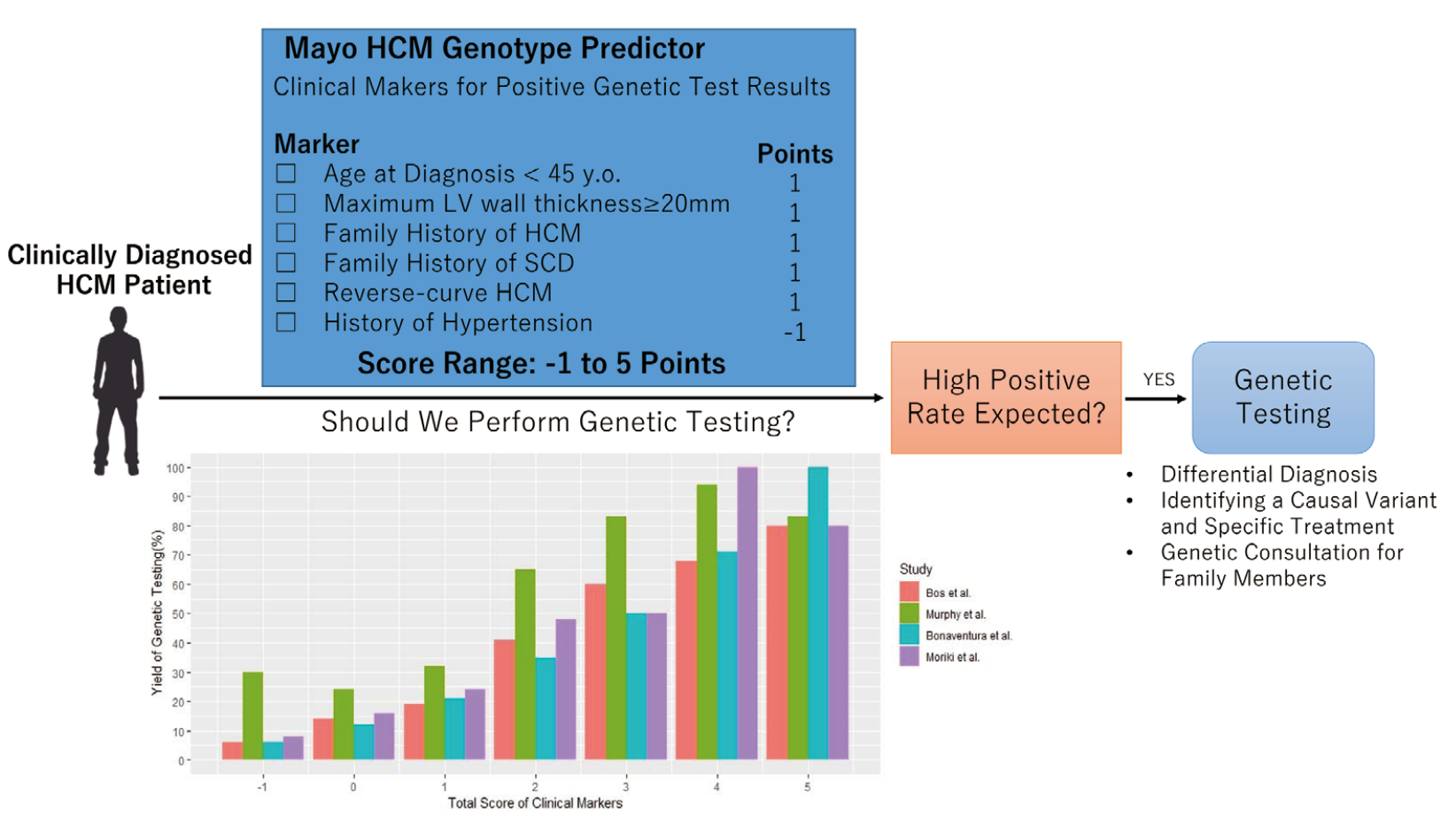

Figure. The Mayo HCM genotype predictor comprises 6 clinical markers, each of which is assigned -1 or +1 for a total score of -1 to +5 . The bar graph shows the yield of genetic testing according to the score in 4 relevant studies (references $1,4,5,6$, respectively). HCM, hypertrophic cardiomyopathy; LV, left ventricular; SCD, sudden cardiac death.

The opinions expressed in this article are not necessarily those of the editors or of the Japanese Circulation Society. Received December 21, 2020; accepted December 21, 2020; J-STAGE Advance Publication released online January 30, 2021 Laboratory for Cardiovascular Genomics and Informatics, RIKEN Center for Integrative Medical Sciences, Yokohama (K.I.); Department of Cardiovascular Medicine, Graduate School of Medicine, The University of Tokyo, Tokyo (H.M.), Japan

Mailing address: Kaoru Ito, MD, PhD, Laboratory for Cardiovascular Genomics and Informatics, RIKEN Center for Integrative Medical Sciences, 1-7-22 Suehiro-cho, Tsurumi-ku, Yokohama 230-0045, Japan. E-mail: kaoru.ito@riken.jp

All rights are reserved to the Japanese Circulation Society. For permissions, please e-mail: cj@j-circ.or.jp ISSN-1346-9843 
has a positive rate of approximately $30^{\frac{1}{1,2}}$ when examining HCM-relevant genes. These positive rates are expected to increase when whole-exome sequencing or wholegenome sequencing is performed or when the "variant of unknown significance" problem has been solved, but the positive rates are still not high.

Therefore, it would be beneficial for both medical professionals and patients if some of the clinical manifestations of HCM could be used as an indicator of whether or not to perform genetic testing, which would improve the yield of genetic testing. For this purpose, the Toronto HCM genotype score was proposed by Gruner et al in 2013. ${ }^{3}$ This score is based on several clinical markers such as age, sex, hypertension, family history, and echocardiographic measurements. But the points value assigned to each clinical marker varies from -7 to +6 , which makes the score complicated to calculate in actual clinical settings. In addition, the significance of being female, incorporated into this scoring system, was later denied. ${ }^{1}$ In 2014, Bos et al developed the Mayo HCM genotype predictor (hereafter referred to as the Mayo score) by simply assigning a point of -1 or +1 to each of 6 clinical markers ${ }^{1}$ (Figure). According to their report, the rate of positive genetic tests increases as this score increases, with the highest score of 5 indicating a positive genetic test rate of up to $80 \%$. Murphy et al validated the relationship between the Mayo score and positive genetic test rate in a group of 564 clinically diagnosed HCM patients, and found that $94 \%$ of patients with a score of 4 had a positive genetic test, and $83 \%$ of those with the highest score of 5.4 In addition, although the number of genes tested was expanded from 9 to 229 using panel sequencing in a study conducted by Bonaventura et al, 5 the same trend as in the original Mayo study ${ }^{1}$ was observed in the association between the Mayo score and the positive genetic test rate, where the highest score of 5 corresponded to a positive genetic test rate of $100 \%$. Thus, the Mayo score was proven to be useful even if the number of HCM genes tested was expanded, suggesting that the Mayo score should be valid even if the number of HCM-related genes increases with the spread of whole-genome sequencing in the near future.

However, whether such a scoring system established for Europeans can be directly applied to other ethnic groups requires careful consideration. In particular, because Europeans and Japanese may have different environmental factors, genetic backgrounds and pathological phenotypes, it is necessary to carefully examine whether the Mayo score can be directly applied to the Japanese.

In this issue of the Journal, Moriki et $\mathbf{a l}^{\mathbf{6}}$ examine the correlation between the Mayo score and a positive genetic test rate in 209 clinically diagnosed HCM patients in order to verify the performance of the Mayo score in Japanese patients with HCM. Similar to the report by Bonaventura et al, $\mathbf{5}$ the tendency for a positive genetic test rate to increase as the score increased was confirmed, highlighting that the Mayo score is useful also in Japanese. Thus, the Mayo score could be useful beyond the ethnic differences between Europeans and Asians. In addition, although 9 sarcomere genes were analyzed in the original study for the Mayo score, ${ }^{1}$ Moriki et al could replicate the effectiveness of Mayo score with screening of only 6 sarcomere genes, indicating that the Mayo score could be a robust prediction score even if the number of genes tested is altered.

The use of such a prediction score for HCM makes genetic testing more efficient and thus increases the likelihood of obtaining useful information. Genetic testing not only helps to identify the causative variant for HCM, but also differentiates HCM from the other diseases with similar phenotypes. For example, cardiac amyloidosis, Fabry disease, glycogenosis, and mitochondrial cardiomyopathy may show a HCM-like cardiac manifestation. Tafamidis meglumine was approved in 2019 for the treatment of transthyretin cardiac amyloidosis, ${ }^{7}$ and enzyme replacement therapy is available for Fabry disease and glycogenosis type II. Differentiating these diseases has great value in terms of choosing and providing a specific treatment. On the other hand, it has been reported that patients with sarcomere mutations have a worse prognosis than those without sarcomere mutations and family history. ${ }^{8} \mathrm{~A}$ direct sarcomere inhibitor, mavacamten, has been recently developed and the phase III clinical trial in patients with obstructive HCM, EXPLORER-HCM, has demonstrated its efficacy and safety. ${ }^{2}$ Thus, genetic testing of patients with HCM can not only clarify the etiology of the disease and determine the need for follow-up and screening of family members, but also provide an opportunity to choose the upcoming specific treatment. Currently in clinical practice, genetic testing is only available at a limited number of facilities, even for patients diagnosed with HCM. In the near future, the number of hospitals and facilities that offer genetic testing will increase and the benefits that patients can receive from the genetic testing will become clearer. A prediction score for positive genetic test is expected to be a powerful tool in the promotion of genetic testing for HCM.

\section{Disclosures}

Conflicts of Interest: None declared. Grants: None applicable. H.M. is a member of Circulation Journal's Editorial Team.

\section{References}

1. Bos JM, Will ML, Gersh BJ, Kruisselbrink TM, Ommen SR, Ackerman MJ. Characterization of a phenotype-based genetic test prediction score for unrelated patients with hypertrophic cardiomyopathy. Mayo Clin Proc 2014; 89: 727-737.

2. Olivotto I, Oreziak A, Barriales-Villa R, Abraham TP, Masri A, Garcia-Pavia P, et al; EXPLORER-HCM study investigators. Mavacamten for treatment of symptomatic obstructive hypertrophic cardiomyopathy (EXPLORER-HCM): A randomised, double-blind, placebo-controlled, phase 3 trial. Lancet 2020; 396: $759-769$.

3. Gruner C, Ivanov J, Care M, Williams L, Moravsky G, Yang H, et al. Toronto hypertrophic cardiomyopathy genotype score for prediction of a positive genotype in hypertrophic cardiomyopathy. Circ Cardiovasc Genet 2013; 6: 19-26.

4. Murphy SL, Anderson JH, Kapplinger JD, Kruisselbrink TM, Gersh BJ, Ommen SR, et al. Evaluation of the Mayo Clinic phenotype-based genotype predictor score in patients with clinically diagnosed hypertrophic cardiomyopathy. $J$ Cardiovasc Transl Res 2016; 9: 153-161.

5. Bonaventura J, Norambuena $\mathrm{P}$, Tomašov $\mathrm{P}$, Jindrová $\mathrm{D}$, Šedivá $\mathrm{H}$, Macek M Jr, et al. The utility of the Mayo Score for predicting the yield of genetic testing in patients with hypertrophic cardiomyopathy. Arch Med Sci 2019; 15: 641-649.

6. Moriki T, Kubo T, Sugiura K, Ochi Y, Baba Y, Hirota T, et al. A validation study of the Mayo Clinic phenotype-based genetic test prediction score for Japanese patients with hypertrophic cardiomyopathy. Circ J 2021; 85: 669-674.

7. Kazi DS, Bellows BK, Baron SJ, Shen C, Cohen DJ, Spertus JA, et al. Cost-effectiveness of tafamidis therapy for transthyretin amyloid cardiomyopathy. Circulation 2020; 141: 1214-1224.

8. Ingles J, Burns C, Bagnall RD, Lam L, Yeates L, Sarina T, et al. Nonfamilial hypertrophic cardiomyopathy: Prevalence, natural history, and clinical implications. Circ Cardiovasc Genet 2017; 10: $\mathrm{e} 001620$ 